\title{
Jogos eletrônicos na atenção à saúde de crianças e adolescentes: revisão integrativa
}

\author{
Electronic games in child and adolescent health care: an integrative review \\ Juegos electrónicos en la atención en salud de niños y adolescentes: revisión integradora
}

Isabelle de Araújo Brandão ${ }^{1}$

Maria Carolina Ortiz Whitaker ${ }^{1}$

Márcia Maria Carneiro Oliveira ${ }^{1}$

Andrea Broch Siqueira Lusquinhos Lessa ${ }^{1}$

Tamily Freitas Santos Lopes ${ }^{1}$

Climene Laura de Camargo ${ }^{1}$

Josielson Costa da Silva ${ }^{1}$

Jessica Alves Sacramento de Moraes ${ }^{1}$

\section{Descritores}

Criança hospitalizada; Jogos de vídeo; Jogos e brinquedos; Promoção da saúde; Doença crônica

\section{Keywords}

Child, hospitalized; Video games; Play and playthings; Health promotion; Chronic disease

\section{Descriptores}

Niño hospitalizado; Juegos de vídeo; Juego e implementos de juego; Promoción de la salud; Enfermedad crônica

\section{Submetido}

12 de Novembro de 2018

\section{Aceito}

2 de Maio de 2019

\section{Autor correspondente}

Maria Carolina Ortiz Whitaker

https://orcid.org/0000-0003-0253-3831

Email: maria.ortiz@ufba.br

\section{DOI}

http://dx.doi.org/10.1590/1982-

0194201900063

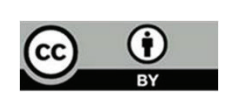

\section{Resumo}

No contexto atual encontramos brincadeiras que envolvem tecnologias avançadas, como o uso de notebooks, smartphones e videogames, sejam no cenário hospitalar, ambulatorial, em clínicas ou em casa, esses dispositivos inovadores estão inseridos na promoção, prevenção, tratamento e recuperação da saúde. Diante do exposto, este estudo tem como objetivo descrever através de revisão integrativa a utilização de jogos eletrônicos na atenção à saúde da criança e adolescentes. Trata-se de uma revisão da literatura nacional e internacional, dos artigos publicados entre os anos de 2007 a 2017, que abordam as implicacões do uso de jogos eletrônicos. A coleta de dados foi realizada no período entre janeiro e abril de 2018, por meio de consulta na Biblioteca Virtual em Saúde (BVS), selecionando-se as bases de dados (MEDLINE, LILACS e BDENF) utilizando os Descritores em Ciências da Saúde (DeCS), pela Biblioteca Virtual em Saúde (BVS). Foram utilizados os seguintes descritores: "Criança hospitalizada/ Child Hospitalized"; "Jogos de vídeo/Video Games"; "Promoção da saúde/Health Promotion". A análise foi realizada a partir da amostra final com 11 artigos. Diante dos achados, foi possível identificar que a literatura aborda questões relacionadas na utilização de jogos eletrônicos. Temáticas relacionadas com promoção, prevenção, tratamento e recuperação da saúde em situações de doenças crônicas e 0 estímulo de atividades físicas, foram evidenciados no estudo. Com isso, percebe-se a relevância que os profissionais de saúde, em especial os de enfermagem, têm em saber que os jogos eletrônicos podem auxiliar no cuidado de crianças com doenças agudas ou crônicas, que estejam ou não hospitalizadas.

\section{Abstract}

In the current context we find games that involve advanced technologies, such as the use of notebooks, smartphones and video games, whether in the hospital, outpatient, medical clinic, or home setting, these innovative devices are integrated into health promotion, prevention, treatment, and recovery. This study aimed to describe, using an integrative review, the use of electronic games in child and adolescent health care. This was a review of national and international literature; articles published between the years 2007 and 2017, which addresses the implications of electronic game usage. Data collection was performed between January and April of 2018, in the Virtual Health Library (VHL), selecting databases (MEDLINE, LILACS and BDENF) using the VHL Health Sciences Descriptors (DeCS). The following descriptors were used: "Criança hospitalizada/ Hospitalized Child"; "Jogos de vídeo/Video Games"; "Promoção da saúde/Health Promotion". The analysis was performed from the final sample of 11 articles. The literature addresses issues related to the use of electronic games. Issues related to health promotion, prevention, treatment and recovery in situations of chronic diseases, and the stimulation of physical activities were evidenced in the study. This findings showed the relevance for health professionals, especially nurses, to know that electronic games can help in the care for children with acute or chronic diseases, whether or not they are hospitalized.

\section{Resumen}

En el contexto actual, encontramos juegos que incluyen tecnologías avanzadas, como el uso de notebooks, smartphones y videojuegos. Ya sea en el escenario hospitalario, ambulatorio, en clínicas o en casa, estos dispositivos innovadores están incorporados en la promoción, prevención, tratamiento y recuperación de la salud. Ante lo expuesto, este estudio tiene el objetivo de describir, a través de revisión integradora, la utilización de juegos electrónicos en la atención en salud de niños y adolescentes. Se trata de una revisión de la literatura nacional e internacional de artículos publicados entre 2007 y 2017, que abordan las implicaciones del uso de juegos electrónicos. La recolección de datos se realizó en el período entre enero y abril de 2018, por medio de consulta en la Biblioteca Virtual em Saúde (BVS), donde se seleccionaron las bases de datos (MEDLINE, LILACS y BDENF) y se utilizaron los descriptores en Ciencias de la Salud (DeCS), de la Biblioteca Virtual em Saúde (BVS). Fueron utilizados los siguientes descriptores: "Niño hospitalizado/ Child Hospitalized"; "Videojuegos/Video Games"; "Promoción de la salud/Health Promotion". El análisis fue realizado a partir de la muestra final con 11 artículos. Entre los descubrimientos, fue posible identificar que la literatura aborda cuestiones relacionadas con la utilización de juegos electrónicos. En el estudio se observaron temáticas relacionadas con promoción, prevención, tratamiento y recuperación de la salud en situaciones de enfermedades crónicas y el estímulo de actividades físicas. De esta forma, se percibe la relevancia que los profesionales de la salud, en especial los de enfermería, tienen en saber que los juegos electrónicos pueden ayudar en el cuidado de niños con enfermedades agudas o crónicas, que estén hospitalizados o no.

\section{Como citar:}

Brandão IA, Whitaker MC, Oliveira MM, Lessa AB, Lopes TF, Camargo CL, et al. Jogos eletrônicos na atenção à saúde de crianças e adolescentes: revisão integrativa. Acta Paul Eferm.2019;32(4):464-9. 


\section{Introdução}

É conhecido que frente à necessidade de hospitalização de crianças e adolescentes o lúdico e o brincar, podem ser estratégias de que auxilia a compreensão, na promoção, prevenção, tratamento e na recuperação da saúde. Brincar é atividade própria da infância que está relacionada ao seu crescimento e desenvolvimento tanto motor quanto emocional, mental e social. A brincadeira e os jogos auxiliam na formação pessoal e potencializam a habilidades. ${ }^{(1)}$

Ao longo do tempo, as formas de atividades lúdicas têm-se modificado em virtude da influência do avanço tecnológico que apresenta posicionamentos controversos de profissionais de saúde com destaque aos prejuízos causados pelo uso excessivo de jogos eletrônicos e exposição às mídias digitais. ${ }^{(2)}$ Contudo é inegável que, tem se tornado crescente o número de brinquedos industrializados e digitais. É reconhecido que atualmente crianças e adolescentes utilizam os equipamentos eletrônicos como forma de diversão, e que esses objetos (televisão, computador, jogos e softwares) são mencionados por elas pela facilidade de manuseio, pois não precisam de grandes espaços e atividade física para utilizá-los. ${ }^{(3)}$

A relação de saúde com dispositivos eletrônicos refere-se a tecnologias de comunicação que possam causar uma mudança comportamental, impacto na prática de atividade física e hábitos alimentares. É sinônimo de informática em saúde, abrangendo processos digitais, prática de cuidados online, além de aplicativos e recursos de dispositivos móveis. Envolve serviços como prontuário eletrônico, sistemas de informação, telemedicina, entre outros. Uma das ferramentas de saúde eletrônica é a intervenção eletrônica, que consiste na promoção, prevenção, tratamento e gerenciamento da saúde física e mental, com ou sem o apoio do ser humano, estimulando a criança e o adolescente a terem comportamentos saudáveis e reduzirem o problema de saúde. Dentre as intervençóes, os jogos eletrônicos são eficazes devido à sua atratividade e entretenimento para os usuários, melhorando seus conhecimentos e as habilidades relacionadas aos cuidados de sua própria saúde. ${ }^{(4)}$
Diante do exposto definiu-se como objetivo descrever através de revisão integrativa a utilização de jogos eletrônicos na atenção à saúde da criança e adolescentes.

\section{Métodos}

Trata-se de uma revisão integrativa da literatura, método que permite o levantamento e análise de subsídios na literatura de forma ampla e sistematizada. A revisão integrativa é um método que tem como finalidade sintetizar resultados obtidos em pesquisas sobre um tema ou questão, de maneira ordenada e abrangente. ${ }^{(5)}$

Para tanto, foram percorridas seis etapas: 1 -identificação do problema com definição da questão de pesquisa; 2-busca em base de dados e bibliotecas virtuais por meio de descritores; 3- tabulação dos estudos; 4- leitura individual dos textos completos para a análise crítica em relação à sua aderência ao objetivo desta pesquisa; 5-interpretação dos resultados; e 6- síntese do conhecimento. ${ }^{(6)}$

A primeira etapa foi composta pela identificaçáo do problema com a definiçáa da questáo de pesquisa: quais as evidências científicas mais recentes sobre as implicaçóes do uso de jogos eletrônicos na promoção, prevenção, tratamento e recuperação da saúde de crianças e adolescentes?

A segunda etapa do estudo consistiu na busca de artigos nas bases de dados da Biblioteca Virtual de Saúde (BVS): Literatura Latino-Americana e do Caribe em Ciências da Saúde (LILACS), Siste ma de Análise e Recuperação da Literatura Médica (MEDLINE) e Base de Dados de Enfermagem (BDENF). Delimitou-se como recorte temporal de artigos publicados no período de 2007 a 2017, com o propósito de evidenciar as publicaçóes mais recentes sobre a temática em questão. A busca dos artigos deu-se através dos descritores selecionados, sendo norteada através dos seguintes critérios de inclusão: artigos nos idiomas português e inglês, disponíveis na íntegra, que contemplaram a temática principal, que abordassem sobre jogos de vídeo na promoção, prevenção, tratamento e recuperação da saúde de crianças e adolescentes, disponíveis em texto completo e que abordassem a questão de pesquisa. 


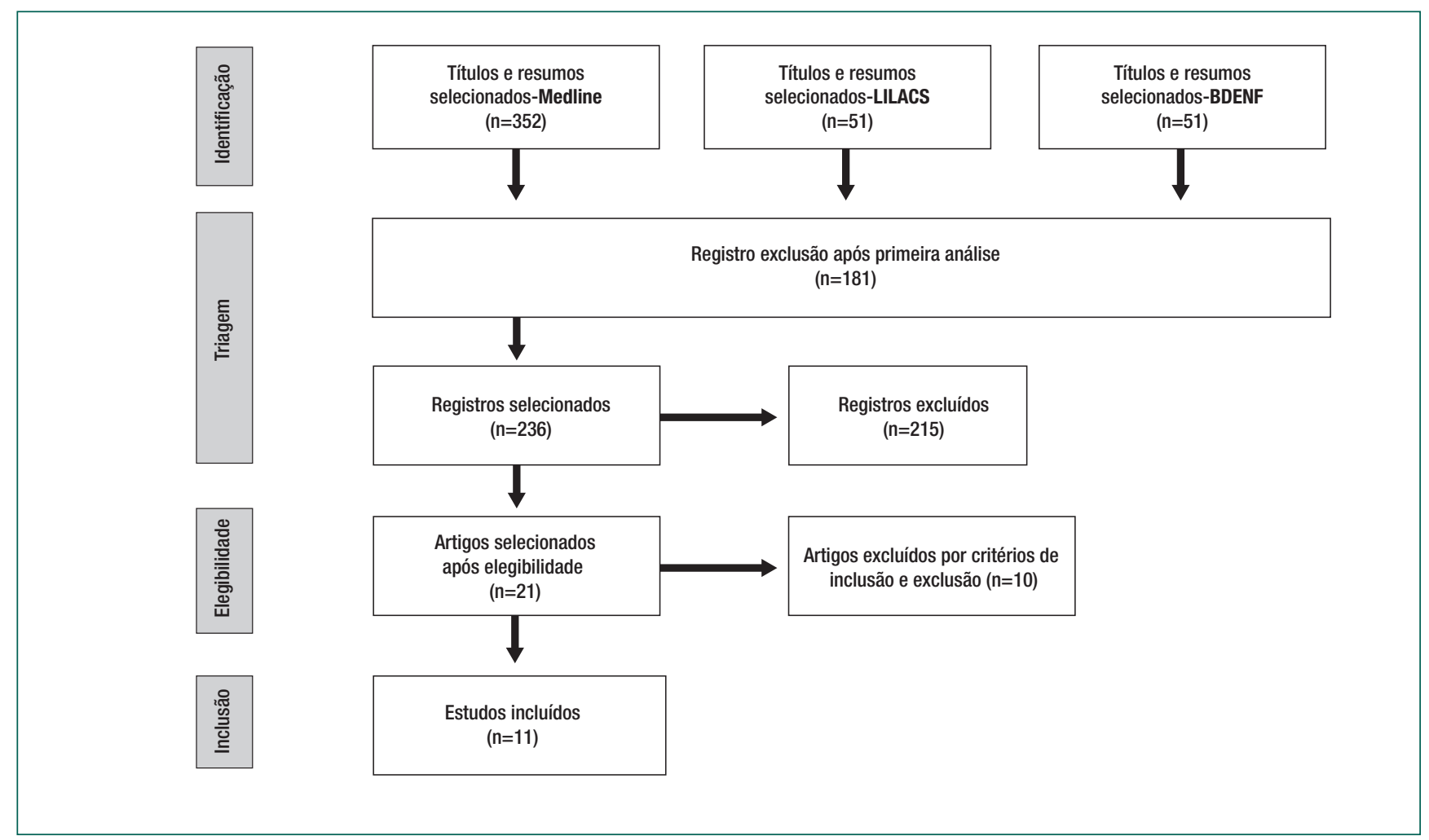

Figura 1. Fluxograma do processo de busca e seleção dos estudos

Como critérios de exclusão não participaram do estudo manuscritos que não respondiam à questão de pesquisa, que analisaram cartas ao editor, duplicatas, estudos do tipo revisão, teses, dissertaçôes, artigos de opiniáo, comentários, ensaios, notas prévias e manuais. A coleta de dados ocorreu no período de janeiro e abril de 2018. O levantamento realizado ocorreu através dos descritores em saúde disponíveis no Portal de Descritores em Ciências da Saúde (DeCS) na BVS.

A seleção dos descritores foi norteada por sua proximidade ao objeto em questão. Chegou-se as seguintes combinaçóes: hospitalized children AND video games; hospitalized children AND health promotion; video games AND health promotion; hospitalized children AND video games AND health promotion.

A terceira etapa consistiu na tabulação dos estudos no Software Microsoft Excel 2010 para organização e sumarização das principais informações, constituindo um banco de dados, possibilitando às pesquisadoras a análise da aplicabilidade da revisão, que contou com as seguintes variáveis: número do artigo $(\mathrm{N})$, título, autores, país do estudo, ano de publicação, tipos de jogos/conclusão, do uso de jogos de vídeo no cuidado da saúde de crianças e adolescentes.
A seguir, foi realizada a quarta etapa composta pela leitura individual dos artigos completos para análise crítica em relação a sua aderência ao objeto desta pesquisa. Após a definição do número final de artigos para compor a revisão, foram realizadas a quinta e sexta etapas, que dizem respeito ao agrupamento dos resultados (evidências) em categorias internamente homogêneas e heterogêneas entre si, para posteriormente ser apresentada a síntese do conhecimento. Após o processo de seleção e identificação dos artigos, foi selecionado 11 artigos, conforme figura 1 .

\section{Resultados}

O quadro 1, apresenta as descrições referente ao título, autores, país, ano de publicação, tipos de jogos e conclusão. Dos 11 artigos incluídos no estudo três foram de 2014, ano com mais publicaçôes, dois destes artigos foram publicados no ano de 2013, assim como nos anos de 2015 e 2016, seguido destes vieram os anos de 2012 e 2011 com apenas um artigo publicado. 
Quadro 1. Artigos selecionados, segundo título, autores, país, ano, tipos de jogos e conclusão

\begin{tabular}{|c|c|c|c|c|}
\hline$N$ & Título & Autores & $\begin{array}{l}\text { País do estudo } \\
\text { /Ano }\end{array}$ & Tipos de jogos/ Conclusão \\
\hline 01 & $\begin{array}{l}\text { Play as a care strategy for children } \\
\text { with câncer. }\end{array}$ & Lima KYN, et al. ${ }^{(3)}$ & Brasil/ 2015 & $\begin{array}{l}\text { Tipos de jogos: Jogos de vídeo, uso de televisão, computador, brinquedos convencionais, } \\
\text { desenhos e atividade na brinquedoteca. Conclusão: Tais atividades proporcionam diversão, } \\
\text { sentimentos de alegria, distração e interação com outras pessoas, reduzindo os efeitos estressores } \\
\text { da hospitalização. }\end{array}$ \\
\hline 02 & $\begin{array}{l}\text { Serious game development as a } \\
\text { strategy for health promotion and } \\
\text { tackling childhood obesity. }\end{array}$ & $\begin{array}{l}\text { Dias JD, } \\
\text { et al. }{ }^{(7)}\end{array}$ & $\begin{array}{l}\text { Brasil/ } \\
2016\end{array}$ & $\begin{array}{l}\text { Tipos de jogos: serious game com jogos eletrônicos sobre alimentação saudável, prática de } \\
\text { exercícios físicos e mudanças nos hábitos de vida. Conclusão: grande potencial educacional } \\
\text { (recurso tecnológico) que pode ser utilizado para o público infantil em escolas e unidades de saúde }\end{array}$ \\
\hline 03 & $\begin{array}{l}\text { Diabetic Mario: designing and } \\
\text { Evaluating Mobile Games for Diabetes } \\
\text { Education. } \\
\end{array}$ & $\begin{array}{l}\text { Baghaei N, et } \\
\text { al. } .^{(8)}\end{array}$ & Nova Zelandia/2016 & $\begin{array}{l}\text { Tipos de jogos: dispositivos móveis sobre dieta e estilo de vida saudáveis. Conclusão: } 0 \\
\text { conhecimento relacionado à diabetes através do jogo pode vir a facilitar a conversa das crianças } \\
\text { com os profissionais de saúde sobre suas práticas de autogestão. }\end{array}$ \\
\hline 04 & $\begin{array}{l}\text { Preventing Adolescents' Diabesity: } \\
\text { Design, Development, and First } \\
\text { Evaluation of "Gustavo in Gnam's } \\
\text { Planet" }\end{array}$ & $\begin{array}{l}\text { Marchetti D, } \\
\text { et al. } .^{(9)}\end{array}$ & $\begin{array}{l}\text { Italy/ } \\
2015\end{array}$ & $\begin{array}{l}\text { Tipos de jogos: jogos online sobre dieta saudável e prevenção de doenças metabólicas. } \\
\text { Conclusão: Jogos sobre saúde pode ser eficaz na infância e adolescência. Com aumento do } \\
\text { conhecimento nutricional possibilidades de alteração no comportamento alimentar. }\end{array}$ \\
\hline 05 & $\begin{array}{l}\text { Novel methods to collect meaningful } \\
\text { data from adolescents for the } \\
\text { development of health interventions }\end{array}$ & Hieftje K, et al. ${ }^{(10)}$ & 2014 & $\begin{array}{l}\text { Tipos de jogos: jogos de vídeo para a orientação de doenças sexualmente transmissíveis para } \\
\text { adolescentes. Conclusão: Jogos de vídeo (Storytelling Using Graphic lllustration, My Life e Photo } \\
\text { Feedback Project), são ferramentas eficazes para o desenvolvimento de intervenções de saúde } \\
\text { com adolescentes. }\end{array}$ \\
\hline 06 & $\begin{array}{l}\text { Active Video Game Play in Children With } \\
\text { Cerebral Palsy: Potential for Physical } \\
\text { Activity Promotion and Rehabilitation } \\
\text { Therapies }\end{array}$ & $\begin{array}{l}\text { Howcroft J, et } \\
\text { al. }{ }^{(11)}\end{array}$ & Canadá/2012 & $\begin{array}{l}\text { Tipos de jogos: jogos de vídeos game ativos (AVGs) para estimular o aprendizado motor e } \\
\text { reeducação neuromuscular em crianças com paralisia cerebral. Conclusão: AVGs podem ser } \\
\text { estrategicamente elecionados para abordar metas terapêuticas específicas. }\end{array}$ \\
\hline 07 & $\begin{array}{l}\text { Video Game Play, Child Diet, and } \\
\text { Physical Activity Behavior Change: }\end{array}$ & $\begin{array}{l}\text { Baranowsky T, et } \\
\text { al. (12) }\end{array}$ & $\begin{array}{l}\text { Canadá/ } \\
2011\end{array}$ & $\begin{array}{l}\text { Tipos de jogos: épicas de videogames, comparáveis aos jogos de vídeo de qualidade comercial } \\
\text { sobre diabetes. Conclusão: crianças que jogaram esses videogames aumentaram o consumo de } \\
\text { frutas e vegetais por dia, mas não aumentaram o consumo de água e atividade física. }\end{array}$ \\
\hline 08 & $\begin{array}{l}\text { Mixed Reality Virtual Pets to Reduce } \\
\text { Childhood Obesity }\end{array}$ & Johnsen, et al..$^{(13)}$ & Estados Unidos/2014 & $\begin{array}{l}\text { Tipos de jogos: } 0 \text { jogo com um pet virtual que foi projetado em torno do conceito de uma clínica } \\
\text { de animais virtual onde animais de estimação obesos precisavam de exercícios físicos. Conclusão: } \\
\text { Acredita-se que essas recompensas são mais propensas a promover atividades físicas futuras e } \\
\text { auto motivadas levando a criança a ter pesos mais saudáveis. }\end{array}$ \\
\hline 09 & $\begin{array}{l}\text { An investigation of the impact of regular } \\
\text { use of the Wii Fit to improve motor and } \\
\text { psychosocial outcomes in children with } \\
\text { movement difficulties: a pilot study. }\end{array}$ & $\begin{array}{l}\text { Hammond J, et } \\
\text { al. }{ }^{(14)}\end{array}$ & $\begin{array}{l}\text { Reino Unido/ } \\
2014\end{array}$ & $\begin{array}{l}\text { Tipos de jogos: jogos de games ativos que se concentram no equilibrio e na coordenação. } \\
\text { Conclusão: o uso dos jogos de equilibrio por } 10 \text { minutos, três vezes por semana, em uma } \\
\text { configuração escolar durante um período de } 1 \text { mês, pode levar a criança a ganhos na proficiência } \\
\text { motora, percepção sobre sua habilidade motora e o seu bem-estar emocional. }\end{array}$ \\
\hline 10 & $\begin{array}{l}\text { Using a robot to personalise health } \\
\text { education for children with diabetes } \\
\text { type 1: a pilot study }\end{array}$ & $\begin{array}{l}\text { Blanson } \\
\text { Henkemans OA, } \\
\text { et al. } .^{(15)}\end{array}$ & $\begin{array}{l}\text { Países Baixo/ } \\
2013\end{array}$ & $\begin{array}{l}\text { Tipos de jogos: robô humanoide autônomo e programável que contribui para o conhecimento do } \\
\text { diabetes infantil. Conclusão: uso das tecnologias da informação está positivamente relacionado } \\
\text { aos altos níveis de habilidade e controle; altos níveis de desafio, diversão e excitação; motivação, } \\
\text { atenção e engajamento focados. }\end{array}$ \\
\hline 11 & $\begin{array}{l}\text { Introduction of Active Video Gaming } \\
\text { Into the Middle School Curriculum as } \\
\text { a School-Based Childhood Obesity } \\
\text { Intervention }\end{array}$ & Quinn $\mathrm{M}$, et al. ${ }^{(16)}$ & 2013 & $\begin{array}{l}\text { Tipos de jogos: os jogos virtuais ativos de dança como intervenção, para tornar os estudantes } \\
\text { mais ativos na aula de educação física. Conclusão: } 0 \text { uso dos jogos sugere que os comportamentos } \\
\text { de modelagem positivos propostos na escola podem encorajar comportamentos positivos em casa. }\end{array}$ \\
\hline
\end{tabular}

\section{Discussão}

Analisando os resultados da amostra, foi possível identificar que a literatura aborda questóes relacionadas ao uso dos jogos eletrônicos e atividades lúdicas que auxiliam na promoção, prevenção, tratamento e na recuperação da saúde de crianças e adolescentes, o que possibilitou a definição de duas categorias: jogos eletrônicos no cuidado relacionado às doenças crônicas e jogos eletrônicos no cuidado relacionado à atividade física.

\section{$1^{\text {a }}$ categoria: jogos eletrônicos no cuidado relacionado às doenças crônicas}

Para composiçáo dessa categoria, foram utilizados os estudos de números 01, 02, 03, 04, 07 e 10. Em nossa revisão foram identificados artigos que atra- vés de jogos eletrônicos buscavam a promoção e prevenção relacionadas à alimentação saudável ${ }^{(7-9)} \mathrm{e}$ controle do diabetes melittus $(D M) .{ }^{(12,15)}$ A obesidade infantil e o DM ganharam reconhecimento nacional e internacional como um problema de saúde publica e uma ameaça ao futuro de nossa população. Diante disso o resultado do nosso estudo mostra relevância com uso da tecnologia de jogos eletrônicos, que através da educação em saúde buscam melhorar controle dessas morbidades e menores complicaçóes macro e microvasculares futuras.

Foram identificadas evidências científicas sobre a eficácia de jogos de computador e dinâmicas para promoção da saúde, como educação em saúde bucal e hábitos alimentares; para prevenção de doenças e agravos, como a prevenção da obesidade e do câncer de pele; e para o manejo de uma doença crônica, 
como a asma. Além disso, aborda-se que os jogos melhoram também o funcionamento cognitivo da criança, podendo ser utilizados no treinamento da memória, desenvolvimento de habilidades analíticas e estratégicas. ${ }^{(16,17)}$

Estudo realizado na Nova Zelândia revelou que os pacientes que utilizaram jogos eletrônicos compreenderam mais sobre dieta e estilo de vida saudáveis, e também informaram que o indicador de açúcar no sangue era de fácil compreensão, apontando que o jogo os ajudou a entender melhor as mudanças do nível de açúcar no sangue a partir de suas escolhas. ${ }^{(8)} \mathrm{O}$ conhecimento relacionado à própria condição médica através do jogo descomplica a conversa das crianças com os profissionais de saúde, desenvolvendo nelas o senso das práticas de autogestão.

Reforçando esse achado, pesquisadores referem que os profissionais de saúde, que utilizaram o brincar como recurso de negociação das abordagens que retratavam o modelo biomédico na assistência à criança hospitalizada, indicou que o método é facilitador no processo de trabalho para lidar com o sofrimento. ${ }^{(18)}$ Nesse sentido, o uso de um brinquedo eletrônico robô, pode favorecer a percepçáo de crianças ao assimilar conhecimento sobre $D M$. Esse estudo procurou avaliar a eficácia de intervençôes lúdicas como mediadores na educação em saúde, promovendo a aprendizagem e mudança de comportamentos. ${ }^{(15)}$

\section{$2^{\mathrm{a}}$ categoria: jogos eletrônicos no cuidado relacionado à atividade física}

Para composição dessa categoria, foram utilizados os estudos de números 02, 06, 07, 08, 09 e 11. Já é conhecido na literatura que a prática de exercícios físicos melhora a circulação e previne doenças em crianças e que o avanço da tecnologia e uso de celulares podem deixar crianças com propensão ao sedentarismo. ${ }^{(2)}$

Estudo revelou que há uma diversidade de serious games desenvolvidos na saúde que estimulam a prática de atividade física e apresentam potencial para aumentar o aprendizado motor e melhora a reabilitação física. ${ }^{(11,19)}$ Nesse aspecto, buscou-se verificar o uso do vídeo game como estratégia de prática de atividades motoras complexas em terapia convencional de fisioterapia com crianças com paralisia cerebral", comprovou que o vídeo game permite aumentar os níveis de atividade, melhora da confiança, relaçóes sociais e habilidades. $\mathrm{O}$ jogo de vídeo contribui para o desenvolvimento e manutenção da função cognitiva e terapia física à medida que aperfeiçoa os cuidados da saúde da criança com avanço do desempenho motor através do controle postural e equilíbrio, agilidade de movimentos e habilidades comunicativas. ${ }^{(20)}$

Contrapondo que os jogos eletrônicos podem deixar as crianças mais sedentárias, foi realizada intervenção através de jogos com dança e concluíram que essa atitude adotada nas escolas podem encorajar comportamentos positivos em casa. ${ }^{(16)}$ Diante disso, devemos encorajar crianças e adolescentes na era digital a promover estilos de vida saudável por meio de jogos eletrônicos, associando o desejo da brincadeira a necessidade da atividade física. Essas atitudes podem provocar mudanças nas relações, no comportamento e na rotina de todas as pessoas, identificar os possíveis benefícios que esses dispositivos podem trazer para a atenção à saúde é imprescindível para que os profissionais utilizem esses recursos como estratégias para açôes de promoção, prevenção, tratamento e recuperação junto às crianças e adolescentes.

\section{Considerações finais}

A construção do presente estudo permitiu compreender as implicaçôes do uso de jogos eletrônicos que vêm atualmente influenciando na promoção, prevenção, tratamento e recuperação de crianças e adolescentes. A maioria dos artigos tratou sobre promoção e prevenção de doenças crônicas, principalmente o diabetes mellitus e obesidade. No tratamento e recuperação da saúde destacaram-se o uso de jogos eletrônicos que estimulassem a realização $\mathrm{da}$ atividade física. Identificamos um estudo sobre DST para a população de adolescentes. Essa revisão contribui para que profissionais da saúde estejam vigilantes na assistência à criança e ao adolescente quanto ao uso das mais novas tecnologias para 
que sua condição de saúde/doença seja melhorada ou restaurada com os recursos disponíveis no atual cenário contemporâneo. Foi identificada a importância da equipe multiprofissional, principalmente da enfermeira em várias ocasióes enquanto a criança está em regime de internamento hospitalar, onde a mesma tem o papel de educadora com os brinquedos terapêuticos, incentivadora para que se brinque na brinquedoteca disponível da unidade e motivadora da interação entre as crianças e as famílias.

Essa pesquisa pode oferecer subsídios para outras pesquisas e realização de futuros estudos experimentais em Unidades Pediátricas. Com isso, percebe-se a relevância que os profissionais de saúde, têm em saber que os jogos eletrônicos trazem diversos benefícios para as crianças que estejam ou não hospitalizadas. Reconheçam tais particularidades e se aprofundem teoricamente no tema, se atualizem para que o cuidado prestado seja resolutivo e integral. Como fatores limitantes do estudo, destaca-se a escassez de estudos controlados que avaliem os fatores dos jogos eletrônicos no processo de promoção, prevenção, tratamento e recuperação da saúde de crianças e adolescentes.

\section{Referências}

1. Caleffi CC, Rocha PK, Anders JC, Souza AlJ, Burciaga VB, Serapião LS. Contribuição do brinquedo terapêutico estruturado em um modelo de cuidado de enfermagem para crianças hospitalizadas. Rev Gaúcha Enferm. 2016;37(2):1-8.

2. Sociedade Brasileira de Pediatria. Saúde de crianças e adolescentes na era Digital. São Paulo:Sociedade Brasileira de Pediatria; 2016. [citado 2019 Abr 29]. Disponíve em: http://www.sbp.com.br/src/ uploads/2016/11/19166d-MOrient-Saude-Crian-e-Adolesc.pdf

3. Lima KY, Santos VE. Play as a care strategy for children with cancer. Rev Gaúcha Enferm. 2015;36(2):76-81.

4. Karami M, Hafizi N.[E-game in healthcare: As an E-intervention to promote public health]. Iran J Public Health . 2016; 45(12):1662-4.

5. Ercole FF, Melo LS de, Alcoforado CL. Revisão integrativa versus revisão sistemática. Reme Rev Min Enferm. 2014;18(1):9-11.
6. Mendes KD, Silveira RC, Galvão CM. Revisao integrativa: método de pesquisa para a incorporação de evidências na saúde e na enfermagem. Texto Contexto Enferm. 2008;17(4):758-64.

7. Dias JD, Mekaro MS, Cheng Lu JK, Otsuka JL, Fonseca LM, ZemMascarenhas SH. Serious game development as a strategy for health promotion and tackling childhood obesity. Rev Lat Am Enfermagem. 2016; 24:e2759

8. Baghaei N, Nandigam D, Casey J, Direito A, Maddison R. Diabetic Mario: Designing and evaluating mobile games for diabetes education. Games Health J. 2016; 5(4):270-8.

9. Marchetti D, Fraticelli F, Polcini F, Lato R, Pintaudi B, Nicolucci A, et al. Preventing adolescents' diabesity: design, development, and first evaluation of "Gustavo in Gnam's Planet." Games Health J. 2015; 4(5):344-51.

10. Hieftje K, Duncan LR, Fiellin LE. Novel methods to collect meaningful data from adolescents for the development of health interventions. Health Promot Pract. 2014; 15(5):714-22.

11. Howcroft J, Klejman S, Fehlings D, Wright V, Zabjek K, Andrysek J, et al. Active video game play in children with cerebral palsy: Potential for physical activity promotion and rehabilitation therapies. Arch Phys Med Rehabil. 2012; 93(8):1448-56.

12. Baranowski T, Baranowski J, Thompson D, Buday R, Jago R, Griffith MJ, et al. Video game play, child diet, and physical activity behavior change: a randomized clinical trial. Am J Prev Med. 2011; 40(1):33-8.

13. Johnsen K, Ahn SJ, Moore J, Brown S, Robertson TP, Marable A, et al. Mixed reality virtual pets to reduce childhood obesity. IEEE Trans Vis Comput Graph. 2014;20(4):523-30.

14. Hammond J, Jones V, Hill EL, Green D, Male I. An investigation of the impact of regular use of the Wii Fit to improve motor and psychosocial outcomes in children with movement difficulties: A pilot study. Child Care Health Dev. 2014; 40(2):165-75.

15. Blanson Henkemans OA, Bierman BP, Janssen J, Neerincx MA, Looije $\mathrm{R}$, van der Bosch $\mathrm{H}$, et al. Using a robot to personalise health education for children with diabetes type 1: A pilot study. Patient Educ Couns . 2013; 92(2):174-81.

16. Quinn M. Introduction of active video gaming into the middle school curriculum as a school-based childhood obesity intervention. J Pediatr Heal Care. 2013; 27(1):3-12.

17. Coscrato G, Pina JC, Mello DF. Utilização de atividades lúdicas na educação em saúde: uma revisão integrativa da literatura. Acta Paul Enferm. 2010; 23(2):257-63.

18. Mitre RM, Gomes R.A promoção do brincar no contexto da hospitalização infantil como ação de saúde. Cien Saude Colet . 2004; 9(1):147-54

19. Dias JD, Tibes CM, Fonseca LM, Zem-Mascarenhas SH. Uso de serious games para enfrentamento da obesidade infantil: Revisão integrativa da literatura. Texto Contexto Enferm. 2017; 26(1):1-10.

20. Braccialli LM, Almeida VS, Silva FC, Silva MZ. Vídeo game na escola e na clínica: auxiliar da inclusão. J Res Spec Educ Needs. 2016; 16(1):1078-81. 